\title{
The Maxwell-Schrödinger-Dirac Correspondence in Auto Confined Electromagnetic Fields
}

\author{
J. W. VEGT \\ Fontys University \\ Department of Micro System Technology \\ R1 - 2.99/2.50 \\ Postbox $3475600 \mathrm{AH}$ Eindhoven The Netherlands \\ E-mail: J.W.Vegt@tm.tue.nl
}

\begin{abstract}
The dynamic Equilibrium Equation expresses the conditions for a dynamic equilibrium related to the electromagnetic force densities at any space and time coordinate in an arbitrary electromagnetic field configuration. It has been demonstrated in earlier publications ${ }^{(3,4)}$ that quantum mechanical relationships like the Schrödinger- and the Dirac equations can be presented in a way similar as the Dynamic Equilibrium Equation (3) for auto confined electromagnetic fields, indicated as AEONs ( Auto Confined Electromagnetic Entities) . In this article the auto confinement is discussed of monochromatic electromagnetic fields due to electromagnetic interaction with an infinite lifetime, (AEONs). New physical possible configurations of electromagnetic fields are presented in the last section, which configurations have the possibility to generate, mass, charge ands spin in a stable configuration. It is demonstrated in (11) - (25) that monochromatic electromagnetic self confinements with an infinite lifetime ${ }^{(1,4,5)}$, based on electromagnetic interaction (AEONs), physically may exist and form a fundamental underlaying aspect of any theory, describing the inner structure of subatomic particles. Besides the presented theory would offer a more fundamental explanation for the observed transitions of matter into electromagnetic radiation and opposite transitions .
\end{abstract}

\section{Introduction}

Combining the divergence of the Energy Momentum Tensor and relativity for confined electromagnetic fields leads to a fundamental electromagnetic equivalent for quantum mechanical equations like the Schrödinger- and the relativistic Dirac equation ${ }^{(3,4,13)}$. In the following sections the auto con- 
finements of monochromatic electromagnetic fields, indicated as AEONs, will be discussed, based on the Dynamic Equilibrium Equation. It is demonstrated in earlier publications ${ }^{(3,4)}$ that solutions of the Dynamic Equilibrium Equation for Auto Confined Electromagnetic Fields are also solutions of the quantum mechanical equivalents like the Schrödinger- and the relativistic Dirac equation $^{(3,4,13)}$.

\section{The Dynamic Equilibrium Equation:}

Besides Gravitational Confinement (GEONs) ${ }^{(1)}$ (Gravitational Electromagnetic Entities) a second option for auto confined electromagnetic energy is a confinement due to the dynamic equilibrium of the internal electromagnetic forces in the confinement itself. In a way identical to the way that GEONS are described by the gravitational equilibrium equation ${ }^{(1,7,13,)}$ (the Einstein Maxwell Equations), EEONs (Electro-Magneto-Static Confined Electromagnetic Entities) are described by the Dynamic Equilibrium Equation (3).

The Maxwell tensor ${ }^{(9)}$ equals:

$$
T^{a b}=\frac{1}{\mu_{0}}\left[F_{a c} F^{c b}+\frac{1}{4} \delta_{a b} F_{c d} F^{c d}\right]
$$

in which $F_{a b}$ are the elements of the Maxwell tensor defined by:

$$
F_{a b}=\partial_{b} \varphi_{a}-\partial_{a} \varphi_{b}
$$

The four-vector potential $\varphi_{a}$ is given by $\varphi_{a}=(i \varphi / c, \vec{A})$ :, where $\varphi$ is the electric scalar potential, c the speed of light in vacuum and $\vec{A}$ is the magnetic vector potential. Substituting (2) in (1) results in the Energy Momentum Tensor, of which the Dynamic Equilibrium Equation (3) is derived. The dynamic Equilibrium Equation expresses the conditions for a perfect dynamic equilibrium for the electromagnetic force densities at any space and time coordinate in an arbitrary electromagnetic field configuration and equals: 


$$
\begin{aligned}
& -\frac{1}{c^{2}} \frac{\partial(\vec{E} \times \vec{H})}{\partial t}+\varepsilon_{0} \vec{E}(\nabla \bullet \vec{E})-\varepsilon_{0} \vec{E} \times(\nabla \times \vec{E})+ \\
& \mu_{0} \vec{H}(\nabla \bullet \vec{H})-\mu_{0} \vec{H} \times(\nabla \times \vec{H})=0
\end{aligned}
$$

Any solution of (3) represents an electromagnetic field which is in a perfect equilibrium with its surrounding at any space and time coordinate and fulfills the necessary requirements for the physical possibility of the existence of the electromagnetic field. Under that condition equation (3) expresses the force density of an electromagnetic field on itself and its surrounding in a perfect equilibrium and any electromagnetic field configuration can only exist in this equilibrium ${ }^{(15,16)}$. This implies that any field configuration which is not a solution of (3) cannot physically exist . In a spherical coordinate system with coordinates $\mathrm{r}$ (radius), $\theta$ (azimuthal angle), $\varphi$ (tangential angle) and $t$ as time coordinate, the part of the dynamic Equilibrium Equation, representing the equilibrium for the force densities in the radial direction equals:

$$
\begin{gathered}
\varepsilon_{0} \mu_{0}\left[-r H_{\varphi}(r, \theta, \varphi, t) \frac{\partial}{\partial t} E_{\theta}(r, \theta, \varphi, t)+r H_{\theta}(r, \theta, \varphi, t) \frac{\partial}{\partial t} E_{\varphi}(r, \theta, \varphi, t)+\right. \\
\left.r E_{\varphi}(r, \theta, \varphi, t) \frac{\partial}{\partial t} H_{\theta}(r, \theta, \varphi, t)-r E_{\theta}(r, \theta, \varphi, t) \frac{\partial}{\partial t} H_{\varphi}(r, \theta, \varphi, t)\right]+ \\
\varepsilon_{0}\left[2 E_{R}(r, \theta, \varphi, t)^{2} E_{\theta}(r, \theta, \varphi, t)^{2}-E_{\varphi}(r, \theta, \varphi, t)^{2}+C s c(\theta) E_{\varphi}(r, \theta, \varphi, t) \frac{\partial}{\partial \varphi} E_{R}(r, \theta, \varphi, t)+\right. \\
E_{\theta}(r, \theta, \varphi, t) \frac{\partial}{\partial \theta} E_{R}(r, \theta, \varphi, t)+E_{R}(r, \theta, \varphi, t)\left\{\operatorname{Cot}(\theta) E_{\theta}(r, \theta, \varphi, t)+\right. \\
\left.C s c(\theta) \frac{\partial}{\partial \varphi} E_{\varphi}(r, \theta, \varphi, t)+\frac{\partial}{\partial \theta} E_{\theta}(r, \theta, \varphi, t)+r \frac{\partial}{\partial r} E_{R}(r, \theta, \varphi, t)\right\}- \\
\left.r E_{\theta}(r, \theta, \varphi, t) \frac{\partial}{\partial r} E_{\theta}(r, \theta, \varphi, t)-r E_{\varphi}(r, \theta, \varphi, t) \frac{\partial}{\partial r} E_{\varphi}(r, \theta, \varphi, t)\right]+ \\
\mu_{0}\left[2 H r(r, \theta, \varphi, t)^{2}+\operatorname{Cot}(\theta) H r(r, \theta, \varphi, t) H_{\theta}(r, \theta, \varphi, t) H_{\theta}(r, \theta, \varphi, t)^{2} H_{\varphi}(r, \theta, \varphi, t)^{2}+\right. \\
C s c(\theta) H_{\varphi}(r, \theta, \varphi, t) \frac{\partial}{\partial \varphi} H_{R}(r, \theta, \varphi, t)+C s c(\theta) H r(r, \theta, \varphi, t) \frac{\partial}{\partial \varphi} H_{\varphi}(r, \theta, \varphi, t)+ \\
H_{\theta}(r, \theta, \varphi, t) \frac{\partial}{\partial \theta} H_{R}(r, \theta, \varphi, t)+H_{R}(r, \theta, \varphi, t) \frac{\partial}{\partial \theta} H_{\theta}(r, \theta, \varphi, t)+ \\
r H_{R}(r, \theta, \varphi, t) \frac{\partial}{\partial r} H_{R}(r, \theta, \varphi, t) r H_{\theta}(r, \theta, \varphi, t) \frac{\partial}{\partial r} H_{\theta}(r, \theta, \varphi, t) \\
\left.r H_{\varphi}(r, \theta, \varphi, t) \frac{\partial}{\partial r} H_{\varphi}(r, \theta, \varphi, t)\right]=0
\end{gathered}
$$


The part of the dynamic Equilibrium Equation, representing the equilibrium for the force densities in the azimuthal direction equals:

$$
\begin{aligned}
& \varepsilon_{0} \mu_{0}\left[r H_{\varphi}(r, \theta r \varphi, t) \frac{\partial}{\partial t} E_{R}(r, \theta r \varphi, t)-r H_{R}(r, \theta r \varphi, t) \frac{\partial}{\partial t} E_{\varphi}(r, \theta r \varphi, t)\right. \\
& \left.-r E_{\varphi}(r, \theta r \varphi, t) \frac{\partial}{\partial t} H_{R}(r, \theta r \varphi, t)+r E_{R}(r, \theta r \varphi, t) \frac{\partial}{\partial t} H_{\varphi}(r, \theta r \varphi, t)\right]+
\end{aligned}
$$

$\varepsilon_{0}\left[3 \operatorname{Er}(r, \theta E \varphi, t) E_{\theta}(r, \theta r \varphi, t)+\operatorname{Cot}(\theta) E_{\theta}(r, \theta r \varphi, t)^{2}-\operatorname{Cot}\left(\theta \operatorname{CoE} E_{\varphi}(r, \theta r \varphi, t)^{2}+\right.\right.$

$$
\operatorname{Csc}\left(\theta \operatorname{Cs} E_{\varphi}(r, \theta r \varphi, t) \frac{\partial}{\partial \varphi} E_{\theta}(r, \theta r \varphi, t)-\left(E_{R}(r, \theta r \varphi, t) \frac{\partial}{\partial \theta} E_{R}(r, \theta r \varphi, t)-\right.\right.
$$

$E_{\varphi}(r, \theta r \varphi, t) \frac{\partial}{\partial \theta} E_{\varphi}(r, \theta r \varphi, t)+E_{\theta}(r, \theta r \varphi, t)\left(\operatorname{Csc}(\theta) \frac{\partial}{\partial \varphi} E_{\varphi}(r, \theta r \varphi, t)+\frac{\partial}{\partial \theta} E_{\theta}(r, \theta r \varphi, t)+\right.$

$$
\left.\left.r \frac{\partial}{\partial r} E_{R}(r, \theta r \varphi, t)\right)+r E_{R}(r, \theta r \varphi, t) \frac{\partial}{\partial r} E_{\theta}(r, \theta r \varphi, t)\right]+
$$

$\mu_{0}\left[3 H_{R}(r, \theta r \varphi, t) H_{\theta}(r, \theta r \varphi, t)+\operatorname{Cot}(\theta) \quad H_{\theta}(r, \theta r \varphi, t)^{2}-\operatorname{Cot}\left(\theta o t H_{\varphi}(r, \theta r \varphi, t)^{2}+\right.\right.$

$\operatorname{Csc}\left(\theta s c H_{\varphi}(r, \theta r \varphi, t) \frac{\partial}{\partial \varphi} H_{\theta}(r, \theta r \varphi, t)+\operatorname{Csc}(\theta) H_{\theta}(r, \theta r \varphi, t) \frac{\partial}{\partial \varphi} H_{\varphi}(r, \theta r \varphi, t)-\right.$

$H r(r, \theta r \varphi, t) \frac{\partial}{\partial \theta} H_{R}(r, \theta r \varphi, t)+H_{\theta}(r, \theta r \varphi, t) \frac{\partial}{\partial \theta} H_{\theta}(r, \theta r \varphi, t)-H_{\varphi}(r, \theta r \varphi, t) \frac{\partial}{\partial \theta} H_{\varphi}(r, \theta r \varphi, t)+$

$\left.r H_{\theta}(r, \theta r \varphi, t) \frac{\partial}{\partial r} H_{R}(r, \theta r \varphi, t)+r H_{R}(r, \theta r \varphi, t) \frac{\partial}{\partial r} H_{\theta}(r, \theta r \varphi, t)\right]=0$

The part of the dynamic Equilibrium Equation, representing the equilibrium for the force density in the tangential direction equals:

$$
\begin{gathered}
\varepsilon_{0} \mu_{0}\left[r H_{\theta}(r, \theta, \varphi, t) \frac{\partial}{\partial t} E_{R}(r, \theta, \varphi, t)+r H_{R}(r, \theta, \varphi, t) \frac{\partial}{\partial t} E_{\theta}(r, \theta, \varphi, t)+\right. \\
\left.r E_{\theta}(r, \theta, \varphi, t) \frac{\partial}{\partial t} H_{R}(r, \theta, \varphi, t) r E_{R}(r, \theta, \varphi, t) \frac{\partial}{\partial t} H_{\theta}(r, \theta, \varphi, t)\right]+ \\
\varepsilon_{0}\left[3 \operatorname{Er}(r, \theta, \varphi, t) E_{\varphi}(r, \theta, \varphi, t)+2 \operatorname{Cot}(\theta) E_{\theta}(r, \theta, \varphi, t) E_{\varphi}(r, \theta, \varphi, t)\right. \\
\operatorname{Csc}(\theta) E_{R}(r, \theta, \varphi, t) \frac{\partial}{\partial \varphi} E_{R}(r, \theta, \varphi, t) \operatorname{Csc}(\theta) E_{\theta}(r, \theta, \varphi, t) \frac{\partial}{\partial \varphi} E_{\theta}(r, \theta, \varphi, t)+ \\
\operatorname{Csc}(\theta) E_{\varphi}(r, \theta, \varphi, t) \frac{\partial}{\partial \varphi} E_{\varphi}(r, \theta, \varphi, t)+E_{\varphi}(r, \theta, \varphi, t) \frac{\partial}{\partial \theta} E_{\theta}(r, \theta, \varphi, t)+E_{\theta}(r, \theta, \varphi, t) \frac{\partial}{\partial \theta} E_{\varphi}(r, \theta, \varphi, t)+ \\
\left.r E_{\varphi}(r, \theta, \varphi, t) \frac{\partial}{\partial r} E_{R}(r, \theta, \varphi, t)+r E_{R}(r, \theta, \varphi, t) \frac{\partial}{\partial r} E_{\varphi}(r, \theta, \varphi, t)\right]+ \\
\mu_{\theta}\left[3 H_{R}(r, \theta, \varphi, t) H_{\varphi}(r, \theta, \varphi, t)+2 \operatorname{Cot}(\theta) H_{\theta}(r, \theta, \varphi, t) H_{\varphi}(r, \theta, \varphi, t)\right. \\
\operatorname{Csc}(\theta) H_{R}(r, \theta, \varphi, t) \frac{\partial}{\partial \varphi} H_{R}(r, \theta, \varphi, t) \operatorname{Csc}(\theta) H_{\theta}(r, \theta, \varphi, t) \frac{\partial}{\partial \varphi} H_{\theta}(r, \theta, \varphi, t)+ \\
\operatorname{Csc}(\theta) H_{\varphi}(r, \theta, \varphi, t) \frac{\partial}{\partial \varphi} H_{\varphi}(r, \theta, \varphi, t)+H_{\varphi}(r, \theta, \varphi, t) \frac{\partial}{\partial \theta} H_{\theta}(r, \theta, \varphi, t)+H_{\theta}(r, \theta, \varphi, t) \frac{\partial}{\partial \theta} H_{\varphi}(r, \theta, \varphi, t)+ \\
\left.r H_{\varphi}(r, \theta, \varphi, t) \frac{\partial}{\partial r} H_{R}(r, \theta, \varphi, t)+r H_{R}(r, \theta, \varphi, t) \frac{\partial}{\partial r} H_{\varphi}(r, \theta, \varphi, t)\right]=0
\end{gathered}
$$




\section{Fundamental solutions of the Dynamic Equilibrium Equation}

As an introduction two well known solutions of (4) are given. An exact (simultaneous) solution of the three parts (4-R), (4-A) en (4-T) of the Dynamic Equilibrium Equations in spherical coordinates $\left(\mathrm{r}, \theta,{ }_{-}\right)$describes the electric field of a proton or an electron in radial direction, known as the electric monopole. In terms of equilibrium aspects of the field there is no principal reason for the physical absence of the magnetic monopole. In (5) is the combination presented of an electric and a magnetic monopole which is an exact solution of (4).

$$
\vec{E}(x, y, z, t)=\left(C \frac{1}{r^{2}}, 0,0\right) ; \quad \vec{H}(x, y, z, t)=\left(C \frac{2}{r^{2}}, 0,0\right)
$$

where $\mathrm{C} 1$ and $\mathrm{C} 2$ are arbitrary constants. A second well known example of an exact solution of the Dynamic Equilibrium Equation (4) equals:

$$
\begin{aligned}
& \vec{E}(x, y, z, t)=\left(0, \frac{f(\theta, \varphi)}{r} g\left(r-\frac{t}{\sqrt{\varepsilon_{0} \mu_{o}}}\right), 0\right) \\
& \vec{H}(x, y, z, t)=\sqrt{\frac{\varepsilon_{0}}{\mu_{0}}}\left(0,0, \frac{f(\theta, \varphi)}{r} g\left(r-\frac{t}{\sqrt{\varepsilon_{0} \mu_{o}}}\right)\right)
\end{aligned}
$$

where $f(\theta, \varphi)$ and $g(r, t)$ are arbitrary functions of $\theta, \varphi$ and $r, t$ respectively. The field presentation (6) describes a spherical electromagnetic wave propagating in the radial direction in vacuum with the speed of light $c_{0}=1 / \sqrt{\varepsilon_{0} \mu_{o}}$.

\section{New solutions of the Dynamic Equilibrium Equation}

\subsection{Electromagnetic interaction}

In the following, the possibilities will be described of the auto confinement of electromagnetic radiation due to the internal electromagnetic forces (Auto Confined Electromagnetic Entities (AEONs) in accordance with the concept of Wheeler ${ }^{(1)}$ who introduced Gravitational Electromagnetic Entities 
(GEONs). In Wheeler's concept an auto confinement occurs due to the gravitational fields which are generated by the electromagnetic radiation itself. The principle of AEONs is based on the concept of electromagnetic interaction. Electromagnetic interaction is an aspect, fundamentally required for the theoretical possibility of AEONs. The principles of this concept will be described below.

An exact solution ${ }^{(14)}$ of the Dynamic Equilibrium Equation (4) in spherical coordinates is presented by $(5)$. When $\mathrm{C} 2=0$, the field presentation in (5) describes an electric monopole where the radial component is proportional to $1 / \mathrm{r}^{2}$. A radial field intensity of $\mathrm{C} 1 / \mathrm{r}^{3}$ for the electric field component is clearly not a solution of the DEE (4). However it is possible to create a static electric field in radial direction, proportional to $1 / \mathrm{r}^{3}$ if the disturbance of the equilibrium is compensated by a second static magnetic field, also proportional to $1 / \mathrm{r}^{3}$ and dependent on $\theta$.

$$
\vec{E}(x, y, z, t)=\left(\begin{array}{c}
\sqrt{2} r^{-3} \sin (\theta) \\
0 \\
0
\end{array}\right) ; \vec{H}(x, y, z, t)=\sqrt{\frac{\varepsilon_{0}}{\mu_{0}}}\left(\begin{array}{c}
0 \\
r^{-3} \sin (\theta) \\
0
\end{array}\right)
$$

Equation (7) ${ }^{(14)}$ describes the electromagnetic interaction between the radial electric field and the azimuthal oriented magnetic field component. Both fields cannot exist separately. Only in a simultaneously electromagnetic interaction between both fields, the required equilibrium at any point in space and time will exist A more general presentation of (7) equals ${ }^{(14)}$ :

$$
\left.\begin{array}{l}
\vec{E}(x, y, z, t)=\left(\begin{array}{c}
\frac{C_{1}}{r^{n+2}} \sin (\theta)^{n} \\
0 \\
0
\end{array}\right) ; \\
\vec{H}(x, y, z, t)=\sqrt{\frac{\varepsilon_{0}}{\mu_{0}}}\left(m \sqrt{\frac{n}{(n+1)}} \frac{C_{1}}{r^{n+2} \sin (\theta)^{n}}\right. \\
0
\end{array}\right)
$$



tric field oriented in the positive $\mathrm{z}$-direction, the electromagnetic interaction causes an increase of the speed of light, while a static electric field oriented in the negative z-direction effects a decrease of the speed of light. For $\mathrm{C}_{2}=$ 1 , the propagation speed $c_{v a r}$ in the positive $\mathrm{z}$-direction reduces to zero, which describes the theoretical possibility of confinement of the electromagnetic pulse. This idea was the basic concept for the described confinements in the next paragraph.

\subsection{Confined Electromagnetic Energy}

The electromagnetic confinements described in the next section all are presented in spherical coordinates. The presented confinements in the following paragraphs have a propagation speed zero in the radial direction. They present standing electromagnetic waves in spherical auto confinements.

4.2.1 Confinements of electromagnetic energy with a single singular point

A part of the solutions of the Dynamic Equilibrium Equation (4), in this article presented in spherical coordinates, have singularities in the origin due to the factor $(1 / \mathrm{r})$ in the solutions. These fields can only exist due to the presence of an elementary particle in the origin. The confined fields are classified in types 1-, 2- 3- or 4- with the Poynting vector oriented in the r,$\theta-,-\varphi$ or a complex orientation direction respectively. The Dynamic Equilibrium Equation (4) gives a variety of exact solutions with the Poynting vector oriented in the radial direction. A solution ${ }^{(14)}$ is indicated as confinement 1 with the corresponding electric field intensity in spherical coordinates. For $r \neq 0$ :

\subsubsection{Confinements classification 1}

The field configuration (11) and (12) is a solution ${ }^{(14)}$ of the dynamic equilibrium equation (4) and is indicated as confinement type 1 with the corresponding electric field intensity in spherical coordinates. For $\mathrm{r} \neq 0$ :

$$
\vec{E}(r, \theta, \varphi, t)=\frac{1}{r}\left(\begin{array}{c}
0 \\
-C 1 f(t)+C 2 \sqrt{C 3-f(t)^{2}} \\
C 2 f(t)+C 1 \sqrt{C 3-f(t)^{2}}
\end{array}\right)
$$


and the corresponding magnetic field intensity in spherical coordinates:

$$
\vec{H}(r, \theta, \varphi, t)=\frac{1}{r} \sqrt{\frac{\varepsilon_{0}}{\mu_{0}}}\left(\begin{array}{c}
0 \\
-C 2 f(t)-C 1 \sqrt{C 3-f(t)^{2}} \\
-C 1 f(t)+C 2 \sqrt{C 3-f(t)^{2}}
\end{array}\right)
$$

$\mathrm{C} 1, \mathrm{C} 2$ and $\mathrm{C} 3$ are arbitrary constants and $\mathrm{f}(\mathrm{t})$ is an arbitrary function of time. If we choose: $\mathrm{f}(\mathrm{t})=\mathrm{Cos}(\omega \mathrm{t})$ and $\mathrm{C} 3=1$, confinement 1.1 can be presented as:

$$
\vec{E}(r, \theta, \varphi, t)=\frac{1}{r}\left(\begin{array}{c}
0 \\
-C 1 \operatorname{Cos}(\omega t)+C 2 \operatorname{Sin}(\omega t) \\
C 2 \operatorname{Cos}(\omega t)+C 1 \operatorname{Sin}(\omega t)
\end{array}\right)
$$

and

$$
\vec{H}(r, \theta, \varphi, t)=\frac{1}{r} \sqrt{\frac{\varepsilon_{0}}{\mu_{0}}}\left(\begin{array}{c}
0 \\
-C 2 \operatorname{Cos}(\omega t)-C 1 \operatorname{Sin}(\omega t) \\
-C 1 \operatorname{Cos}(\omega t)+C 2 \operatorname{Sin}(\omega t)
\end{array}\right)
$$

$\mathrm{C} 1, \mathrm{C} 2$ and $\omega$ are arbitrary constants. (13) and (14) represent an confinement with an infinite life time. If we choose $\mathrm{C} 1=0, \mathrm{C} 2$ and $\mathrm{C} 3$ arbitrary and $\mathrm{f}(\mathrm{t})$ $=\operatorname{Cos}(\omega \mathrm{t})$, confinement 1.1 is presented by:

$$
\vec{E}(r, \theta, \varphi, t)=\frac{C_{2}}{r}\left(\begin{array}{c}
0 \\
\sqrt{\left(C_{3}-\operatorname{Cos}(\omega t)^{2}\right.} \\
\operatorname{Cos}(\omega t)
\end{array}\right) ; \vec{H}(r, \theta, \varphi, t)=\frac{C_{1}}{r} \sqrt{\frac{\varepsilon_{0}}{\mu_{0}}}\left(\begin{array}{c}
0 \\
-\operatorname{Cos}(\omega t) \\
\sqrt{\left(C_{3}-\operatorname{Cos}(\omega t)^{2}\right.}
\end{array}\right)
$$

For the confinement presented in (15), the electric charge density $\rho_{E}$ equals:

$$
\rho_{E}=\varepsilon_{0} \frac{C 2}{r^{2}} \sqrt{C_{3}-\cos (\omega t)^{2}} \cot (\theta)
$$


Averaged over a period interval, the electric charge density for e.g. C3 $=2$ equals:

$$
\rho_{E}=1.216 \frac{C 2}{r^{2}} \operatorname{Cot}(\theta)
$$

The magnetic charge density, averaged over a period interval, in this example equals zero.

The Dynamic Equilibrium Equation (4) gives a variety of exact solutions with the Poynting vector oriented in the radial direction. Another option is a similar solution as (11) and (12) but differs in sign of the separate components. For $\mathrm{r} \neq 0$ :

$$
\vec{E}(r, \theta, \varphi, t)=\frac{1}{r}\left(\begin{array}{c}
0 \\
C 1 f(t)+C 2 \sqrt{C 3-f(t)^{2}} \\
-C 2 f(t)+C 1 \sqrt{C 3-f(t)^{2}}
\end{array}\right)
$$

and the corresponding magnetic field intensity in spherical coordinates:

$$
\vec{H}(r, \theta, \varphi, t)=\frac{1}{r} \sqrt{\frac{\varepsilon_{0}}{\mu_{0}}}\left(\begin{array}{c}
0 \\
C 2 f(t)-C 1 \sqrt{C 3-f(t)^{2}} \\
C 1 f(t)+C 2 \sqrt{C 3-f(t)^{2}}
\end{array}\right)
$$

$\mathrm{C} 1, \mathrm{C} 2$ and $\mathrm{C} 3$ are arbitrary constants and $\mathrm{f}(\mathrm{t})$ is an arbitrary function of time. If we choose: $\mathrm{f}(\mathrm{t})=\operatorname{Cos}(\omega \mathrm{t})$ and $\mathrm{C} 3=1$, AEON 1.2 can be presented as:

$$
\vec{E}(r, \theta, \varphi, t)=\frac{1}{r}\left(\begin{array}{c}
0 \\
C 1 \operatorname{Cos}(\omega t)+C 2 \operatorname{Sin}(\omega t) \\
-C 2 \operatorname{Cos}(\omega t)+C 1 \operatorname{Sin}(\omega t)
\end{array}\right)
$$

and 


$$
\vec{H}(r, \theta, \varphi, t)=\frac{1}{r} \sqrt{\frac{\varepsilon_{0}}{\mu_{0}}}\left(\begin{array}{c}
0 \\
C 2 \operatorname{Cos}(\omega t)-C 1 \operatorname{Sin}(\omega t) \\
C 1 \operatorname{Cos}(\omega t)+C 2 \operatorname{Sin}(\omega t)
\end{array}\right)
$$

\subsubsection{Confinements classification 3}

Another solution $^{(14)}$ of (4) presented in (22), is the confinement of electromagnetic waves, with the Poynting vector oriented in the $\varphi$-direction of a sphere, which forms a combination of standing electromagnetic waves. For $r \neq 0$ :

$\vec{E}(r, \theta, \varphi, t)=\left(\begin{array}{c}C l \frac{\operatorname{Sin}(\theta)^{n}}{r^{n+2}} \sqrt{C 2-f(t)^{2}} \\ C l \sqrt{\frac{n}{n+1}} \frac{\operatorname{Sin}(\theta)^{n}}{r^{n+2}} f(t) \\ 0\end{array}\right) ; \vec{H}(r, \theta, \varphi, t)=\sqrt{\frac{\varepsilon_{0}}{\mu_{0}}}\left(\begin{array}{c}-C l \frac{\operatorname{Sin}(\theta)^{n}}{C l} f(t) \\ C l \frac{\operatorname{Sin}(\theta)^{n}}{r^{n+2}} \sqrt{\frac{n}{n+1}} \sqrt{C 2-f(t)^{2}} \\ 0\end{array}\right)$

Both fields have no component in the $\varphi$-direction. $\mathrm{C}_{1}, \mathrm{C}_{2}$ and $\mathrm{n}$ are arbitrary constants and $\mathrm{f}(\mathrm{t})$ is an arbitrary function of time. For $\mathrm{C} 2=1$ and $f(t)=\cos (\omega t)$, equation (22) becomes:

$$
\vec{E}(r, \theta, \varphi, t)=\left(\begin{array}{c}
C l \frac{\operatorname{Sin}(\theta)^{n}}{r^{n+2}} \operatorname{Sin}(\omega t) \\
C l \sqrt{\frac{n}{n+1}} \frac{\operatorname{Sin}(\theta)^{n}}{r^{n+2}} \operatorname{Cos}(\omega t) \\
0
\end{array}\right) ; \vec{H}(r, \theta, \varphi, t)=\left(\begin{array}{c}
-C l \frac{\operatorname{Sin}(\theta)^{n}}{r^{n+2}} \operatorname{Cos}(\omega t) \\
C 1 \sqrt{\frac{n}{n+1}} \frac{\operatorname{Sin}(\theta)^{n}}{r^{n+2}} \operatorname{Sin}(\omega t) \\
0
\end{array}\right)
$$

which is the presentation of a confinement with an infinite life time. For $\mathrm{n}=$ $1, \mathrm{C} 2=1$ and $\mathrm{f}(\mathrm{t})=\sin (\omega \mathrm{t})$, the total electric charge $Q_{E A}$ of the auto confined radiation within two spheres with radii $R_{1}$ and $R_{2}$ respectively equals:

$$
Q_{E A}=-2 \varepsilon_{0} C_{1} \pi\left(\frac{1}{R_{1}}-\frac{1}{R_{2}}\right)
$$


The total magnetic charge $\phi_{\mathrm{MA}}$ between two concentric spheres with radii $\mathrm{R} 1$ and R2 respectively, averaged over a period time interval equals zero.

\subsubsection{Confinements classification 4}

Another solution $^{(14)}$ of (4) presented in (25), is the auto confinement of electromagnetic waves, propagating in a complex orientated $r, \theta, \varphi$ direction and resulting in a complex standing electromagnetic wave. For $r \neq 0$ :

$$
\vec{E}(r, \theta, \varphi, t)=\left(\begin{array}{c}
C 1 \frac{\operatorname{Cos}(\omega t)}{r^{2}} \\
\operatorname{Sin}(\theta) \frac{C 2 \operatorname{Sin}(\omega t)}{r} \\
\operatorname{Sin}(\theta) \frac{C 2 \operatorname{Cos}(\omega t)}{r}
\end{array}\right) ; \vec{H}(r, \theta, \varphi, t)=\sqrt{\frac{\varepsilon_{0}}{\mu_{0}}}\left(\begin{array}{c}
C 1 \frac{\operatorname{Sin}(\omega t)}{r^{2}} \\
\operatorname{Sin}(\theta) \frac{-C 2 \operatorname{Cos}(\omega t)}{r} \\
\operatorname{Sin}(\theta) \frac{C 2 \operatorname{Sin}(\omega t)}{r}
\end{array}\right)
$$

\subsubsection{Confinements of electromagnetic energy in symmetric solutions}

A special classification of solutions of the dynamic equilibrium equation is formed by an equilibrium between the electric and the magnetic repulsive and attractive forces due to a symmetry in the electromagnetic components. These special classifications give solutions for any arbitrary field configuration under the condition of a special required symmetry in the electric and the magnetic field components.

\subsubsection{Confinements in symmetric solutions in classification 1}

In (26) an electromagnetic field configuration is presented in which the electromagnetic field components are in a perfect equilibrium and the Poynting vector is oriented in the radial direction in which $f(r, \theta, \varphi, t)$ is a arbitrary function of the radius $r$, the azimuthal angle $\theta$, the tangential angle $\varphi$ and the time t.C1 and C2 are arbitrary constants. 


$$
\vec{E}(r, \theta, \varphi, t)=\left(\begin{array}{c}
0 \\
\sqrt{\frac{C 1}{r}-f(r, \theta, \varphi, t)^{2}} \\
-\sqrt{\frac{C 2}{r}+f(r, \theta, \varphi, t)^{2}}
\end{array}\right)
$$

$$
\vec{H}(r, \theta, \varphi, t)=\sqrt{\frac{\varepsilon_{0}}{\mu_{0}}}\left(\begin{array}{c}
0 \\
\sqrt{\frac{C 2}{r}-f(r, \theta, \varphi, t)^{2}} \\
\sqrt{\frac{C 1}{r}+f(r, \theta, \varphi, t)^{2}}
\end{array}\right)
$$

\subsubsection{Confinements in symmetric solutions in classification 3}

In (26) an electromagnetic field configuration is presented in which the electromagnetic field components are in a perfect equilibrium and the Poynting vector is oriented in the tangential direction in which $f(r, \theta, \varphi, t)$ is arbitrary function of the radius $r$, the azimuthal angle $\theta$, the tangential angle $\varphi$ and the time t. $\mathrm{C} 1$ and $\mathrm{C} 2$ are arbitrary constants.

$$
\begin{gathered}
\vec{E}(r, \theta, \varphi, t)=\left(\begin{array}{c}
-\operatorname{Cos}(\theta) \sqrt{C 1+f(r, \theta, \varphi, t)} \\
\operatorname{Sin}(\theta) \sqrt{C 1+f(r, \theta, \varphi, t)} \\
0
\end{array}\right) \\
\vec{H}(r, \theta, \varphi, t)=\sqrt{\frac{\varepsilon_{0}}{\mu_{0}}}\left(\begin{array}{c}
-\operatorname{Cos}(\theta) \sqrt{C 1-f(r, \theta, \varphi, t)} \\
\operatorname{Sin}(\theta) \sqrt{C 1-f(r, \theta, \varphi, t)} \\
0
\end{array}\right)
\end{gathered}
$$


4.2.2.3 Confinements in symmetric solutions in classification 4

In (26) an electromagnetic field configuration is presented in which the electromagnetic field components are in a perfect equilibrium in which $\mathrm{f}(\mathrm{r}, \theta, \varphi, \mathrm{t})$ is arbitrary function of the radius $\mathrm{r}$, the azimuthal angle $\theta$, the tangential angle $\varphi$ and the time t. C1 and C2 are arbitrary constants.

$$
\begin{aligned}
& \vec{E}(r, \theta, \varphi, t)=\left(\begin{array}{c}
C 1 \frac{f(r, \theta, \varphi, t)}{r} \\
-\frac{\sqrt{C 2-r^{2} f(r, \theta, \varphi, t)^{2}}}{f(r, \theta, \varphi, t)}
\end{array}\right) \\
& \vec{H}(r, \theta, \varphi, t)=\sqrt{\frac{\varepsilon_{0}}{\mu_{0}}}\left(\begin{array}{c}
-C 1 \frac{\sqrt{C 2-r^{2} f(r, \theta, \varphi, t)^{2}}}{r^{2}} \\
-\frac{\sqrt{C 2-r^{2} f(r, \theta, \varphi, \varphi, t)^{2}}}{r}
\end{array}\right)
\end{aligned}
$$

\subsection{Auto Confined Electromagnetic Entities (AEONs)}

In earlier publications ${ }^{(3,4)}$ the theoretical possibility of Auto Confined Electromagnetic Entities has been described as the possible building material for matter in a more likely way than GEONs ${ }^{(1)}$. It will be clear that any possible configuration for AEONs has to be a solution of (4) in electromagnetic components. It appears that in a mathematical way, AEONs offer the possibility of the existence of auto confined radiation with a rest mass unequal to zero, carrying charge and spin.

\section{Applications of the theory:}

An application of the presented theory could be the shielding of valuable computer networks against harmful low frequent electromagnetic radiation. It is well known that low frequent magnetic fields can easily damage entire computer networks and it is easy to erase the information on an entire 
network with low frequent electromagnetic radiation. For that reason, protection of vast networks will be required in the future. It is almost impossible to protect wide networks with classical shielding materials in a wide frequency range. For that reason an alternative method is necessary.

It has been demonstrated in (10) that due to electromagnetic interaction, the speed of light can be altered by coherent electromagnetic radiation. This means that in theory it is possible to change the speed of propagation and the direction of propagation of intense low frequent radiation by coherent radiation, generated simultaneously. In such a way an electromagnetic shielding, covering the entire network, could be obtained.

Because this theory does not follow from the Maxwell Theory (Maxwell's equations in vacuum only involve divergent free electromagnetic waves) but from a more fundamental theory that implies electromagnetic interaction by non divergence free electromagnetic radiation in vacuum, these applications yet have not been investigated.

\section{Validity of the Dynamic Equilibrium Equation:}

The solutions of the dynamic equilibrium equation (4) cover a wider range than the classical solutions for electromagnetic fields. It will be demonstrated in the following that (4) transforms into the well-known set of four Maxwell Equations for divergence free electromagnetic waves. Equation (4) is split into two parts:

$$
\begin{aligned}
& \vec{E} \times\left[\frac{1}{c^{2}} \frac{\partial \vec{H}}{\partial t}-\varepsilon_{0}(\nabla \times \vec{E})\right]=\varepsilon_{0} \vec{E}(\nabla \bullet \vec{E}) \\
& \vec{H} \times\left[\frac{1}{c^{2}} \frac{\partial \vec{E}}{\partial t}-\mu_{0}(\nabla \times \vec{H})\right]=\mu_{0} \vec{H}(\nabla \bullet \vec{H})
\end{aligned}
$$

The vector functions on the left-hand side of (25) are perpendicular tot the vector functions on the right hand side of (25). The only solutions for these vector functions are the vector functions zero which implies that the electromagnetic field has to be divergence free under that condition and (25) transforms into the set of four Maxwell Equations in the absence of any matter. 


$$
\begin{array}{ll}
\nabla \times \vec{E}=-\frac{\partial \vec{B}}{\partial t} & \nabla \bullet \vec{E}=0 \\
\nabla \times \vec{H}=\frac{\partial \vec{D}}{\partial t} & \nabla \bullet \vec{B}=0
\end{array}
$$

\section{Concluding Remarks}

Because of the required extremely high energy densities of self-confined electromagnetic radiation by gravitational forces (GEONs)at the dimensions of elementary particles, the radiation pressure also will be extremely high and varies in magnitude roughly about $10^{70}\left[\mathrm{~N} / \mathrm{m}^{2}\right]$ for gravitational confinements (GEONs, Wheeler ${ }^{(1)}$ ). For that reason a model, describing the elementary sub-structure of matter in which GEONs are considered to be the building elements is not very likely based on terms of stability conditions. The electromagnetic force however is a much more powerful controlling mechanism to create electromagnetic self confinements on a subatomic scale than the gravitational force. It is demonstrated in (11) - (25) that monochromatic electromagnetic self confinements with an infinite lifetime ${ }^{(1,4,5)}$, based on electromagnetic interaction (AEONs), physically may exist and form a fundamental underlaying aspect of any theory, describing the inner structure of sub-atomic particles. It follows from (11)-(25) that AEONs have the possibility to generate, mass, charge ands spin in a stable configuration. Besides the presented theory would offer a more fundamental explanation for the observed transitions of matter in electromagnetic radiation and opposite transitions .

\section{Acknowledgment}

This work has been made possible by the contributions of many to whom I am indebted and Lynn, Antonia, Julian and my family in particular.

\section{References}

[1] Wheeler J, Geons, Phys. Rev. 97 511(1955).

[2] Elbaz C, On Self-Field Electromagnetic Properties for Extended Material Particles, Phys. Lett. A 127308 (1988). 
[3] Vegt J W, A continuous model of matter based on AEONs, Physics Essays, 8-2, 201 (1995).

[4] Vegt J W, A particle free model of matter based on Electromagnetic Selfconfinement, Les Annales de Louis de Broglie, 20-02, 135 (1994).

[5] Kaku M, Introduction to Superstrings, Springer Verlag, New York (1988).

[6] Barbashov B and Nesterenko V, Introduction to the relativistic string theory, World Scientific Publishing, London (1990).

[7] Arias P, Matter coupling in 16/16 super gravity, Karlsruhe Universität, Karlsruhe (1989).

[8] Yaghjian A D, A classical electro-gravitational model of a point charge with a finite mass, Proc. of the URSI (Int. Union of Radio Science) Int. Symposium on Electromagnetic Theory, 14 - 17, 322 (1989).

[9] Rosser W G V, Classical Electromagnetism via Relativity, (Butterworths, London), p. 134 (1968).

[10] Zaluzny M, , On the optical properties of quasi-two-dimensional electrons, Physica B 128171 (1985).

[11] Petrich W, Behavior of atoms in a compressed magneto-optical trap, Journal of the optical society of America B, 11-8, 1332 (1994).

[12] Ovchinnikov Y B, Resonant light pressure on atoms in a bichromatic standing wave, Optics and Spectroscopy, 76-2, 188 (1994).

[13] Laporte $\mathrm{O}$ and Uhlenbeck G, Application of spinor analysis tot the Maxwell and Dirac Equations, Physical Review, 37, 1380 (1931).

[14] Wolfram Research, Mathematica 4.0, Cambridge University Press (1999).

[15] Barrett T.W. \& Grimes D.M, (Eds) Advances Electromagnetism: Foundations, Theory \& Applications, World Scientific. (Chapters Aldrovandi, de Beauregard and Barrett) (1995).

[16] Mikhailov V.F., Les Annales de Louis de Broglie, 24, 161 (1999).

Manuscript reçu le 8 juillet 1999, modifié le 19 novembre 2001 